\title{
Mathematical model of DNA lesions
}

\section{Belov Oleg}

Joint Institute for Nuclear Reserch

E-mail: demejinr.ru

\section{Boyda Denis}

Far Eastern Federal University

E-mail: boyda_d@mail.ru

\section{Shirmovsky Sergey*}

Far Eastern Federal University

E-mail: shirmovsky@sns.dvfu.ru

\begin{abstract}
The study is focused on the simulation of the physical mechanisms of DNA lesion induction under charged particle irradiation. A model approach is proposed to simulate the processes of charge injection and its further migration within a linear double-stranded DNA molecule hit by a particle track. The study is conducted in two steps. First, we simulate the physics of particle interaction with the water medium surrounding DNA and determine the position of positive charge injection. Then, we investigate hole migration on the basis of the nonlinear dynamics of excitations in DNA. This allows finding the most probable locations of the elementary DNA lesions and estimating the complexity of the resulting clustered damage.
\end{abstract}

XXI International Baldin Seminar on High Energy Physics Problems September 10-15, 2012

JINR, Dubna, Russia

${ }^{*}$ Speaker. 


\section{Introduction}

Studying the physical mechanisms of DNA damage with heavy charged particles is one of the central problems of radiobiology and radiation research [1]. As the DNA is the main biological target for ionizing radiation, its lesions determine many biological endpoints like cell killing, induction of chromosome aberrations and structural gene mutations, initiation of cell transformation, and more. Damaging effects of heavy charged particles result from the combination of their direct and indirect action. DNA lesions caused by the direct action of particles occur as a consequence of molecule atom ionization. Indirect action is based on the production of free radicals, which have extremely high chemical reactivity leading to the induction of structural disorder in DNA.

One of the important mechanisms in both direct and indirect effects is charge migration. This process can be realized after direct charge injection by a charged particle as well as after the interaction of a free radical with DNA. The final DNA lesion type and complexity depend to a large extent on charge migration, which points to the significance of this mechanism. Of special interest is the role of charge migration in the formation of clustered DNA lesions, which are the hardest to repair.

In this study, we are focused on the mathematical modeling of positive charge (hole) migration along DNA determined by the direct action of accelerated heavy ions. Here we investigate this process on the basis of a nonlinear, double-stranded model. A DNA molecule is considered as a quasi-regular chemical structure whose regular part is composed of strictly alternating sugar molecules and phosphate groups making up the sugar-phosphate backbone, while its irregular part is composed of bases. The model approach takes into account the double-stranded nature of DNA, stacking interaction through each DNA strand, the existence of hydrogen bonds between bases, and the separate base positions. To obtain the initial location of a hole, we used a Monte Carlo calculation technique for simulating the particle track structure in combination with a space-filling model of a linear double-stranded DNA.

\section{Model approach}

To study the interaction of a charged particle with a DNA molecule, we have simulated the track structure of a ${ }^{12} \mathrm{C}^{4+}$ accelerated ion with an energy of $3.3 \mathrm{MeV} /$ nucleon in a water volume of $1 \mu \mathrm{m}^{3}$. We have used the Monte Carlo technique and physics approaches implemented in the TRIOL code. Then the space-filling model of the 68 bp DNA molecule has been combined with the obtained ionization positions in water (Fig. 1). The most probable sites of the initial charge locations were determined taking into account the radii of the nucleic acid atoms. Here we do not take into account the influence of the DNA molecule on the physics of particle - water medium interaction. We also do not consider the indirect action of radiation connected with reactive free radical production.

To investigate the further migration of an injected hole, we used a nonlinear, double-stranded model of DNA. In the model the full DNA-charge Hamiltonian $\mathscr{H}$ is determined as a sum of two term:

$$
\mathscr{H}=\mathscr{H}_{C l}+\left\langle\psi\left|\mathscr{H}_{Q}\right| \psi\right\rangle
$$




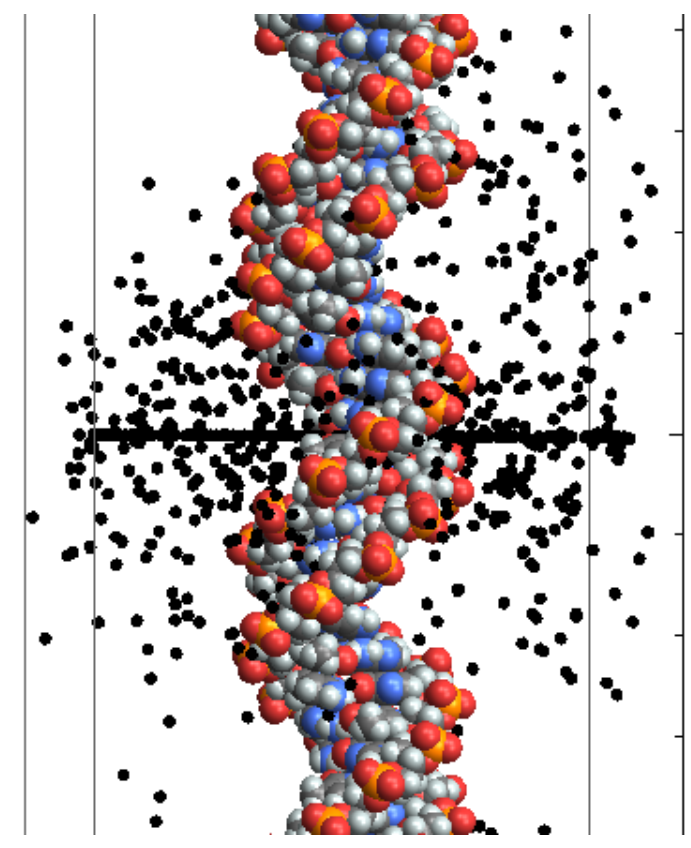

Figure 1: Track structure of a ${ }^{12} \mathrm{C}^{4+}$ ion combined with a space-filling model of DNA.

In this expression the first - classical - part being determined as follows:

$$
\begin{array}{r}
\mathscr{H}_{C l}=\sum_{n=1}^{N}\left[\frac{M \dot{y}_{1, n}^{2}}{2}+\frac{M \dot{y}_{2, n}^{2}}{2}+\frac{k_{v}\left(y_{1, n}-y_{1, n-1}\right)^{2}}{2}+\right. \\
+\frac{k_{v}\left(y_{2, n}-y_{2, n-1}\right)^{2}}{2}+ \\
\left.+\frac{k_{h}\left(y_{1, n}-y_{2, n}+l\right)^{2}}{2}\right] .
\end{array}
$$

with the first and second terms corresponding to the kinetic energy of the bases located on two different DNA strands with the coordinates of $y_{1}$ and $y_{2}$. The bases are coupled together with hydrogen bonds, which are modeled by the fifth term. The third and fourth terms form stacking interaction through each DNA strand. Dot indicates time derivative. The model parameters are: $M$ effective base mass, $k_{h}$ and $k_{v}$ are force constants; $l$ - horizontal distance between the bases in equilibrium. The summation in expression is over the number of base pairs in the strand under consideration. The Hamiltonian presents a DNA macromolecule as an oscillator formed by two flowing strands. The strands are joined to compose DNA (Fig. 2).

The second term in $\mathscr{H}$ expression is determined by a charged particle quantum dynamics. $\mathscr{H}_{Q}$ Hamiltonian determines the properties of the charge located on the bases in the state of $\mid n>(\mid m>)$.

$$
\mathscr{H}_{Q}=\sum_{n=1}^{N} \alpha_{n}^{0}|n\rangle\left\langle n\left|+\sum_{n=1}^{N} \alpha_{n}^{\prime}\left(y_{1, n}-y_{2, n}+l\right)\right| n\right\rangle\left\langle n\left|+\sum_{n \neq m}^{N} v_{n, m}\right| n\right\rangle\langle n|
$$

Here, $\alpha_{n}^{0}$ is equal to an $n$-base charge energy, $v_{n, m}$ are matrix elements of the transition between $n$, $m$ bases. The relationship between the charged particle and DNA in the model is determined by the second component, where $\alpha_{n}^{\prime}$ is a fitting parameter of the model. 


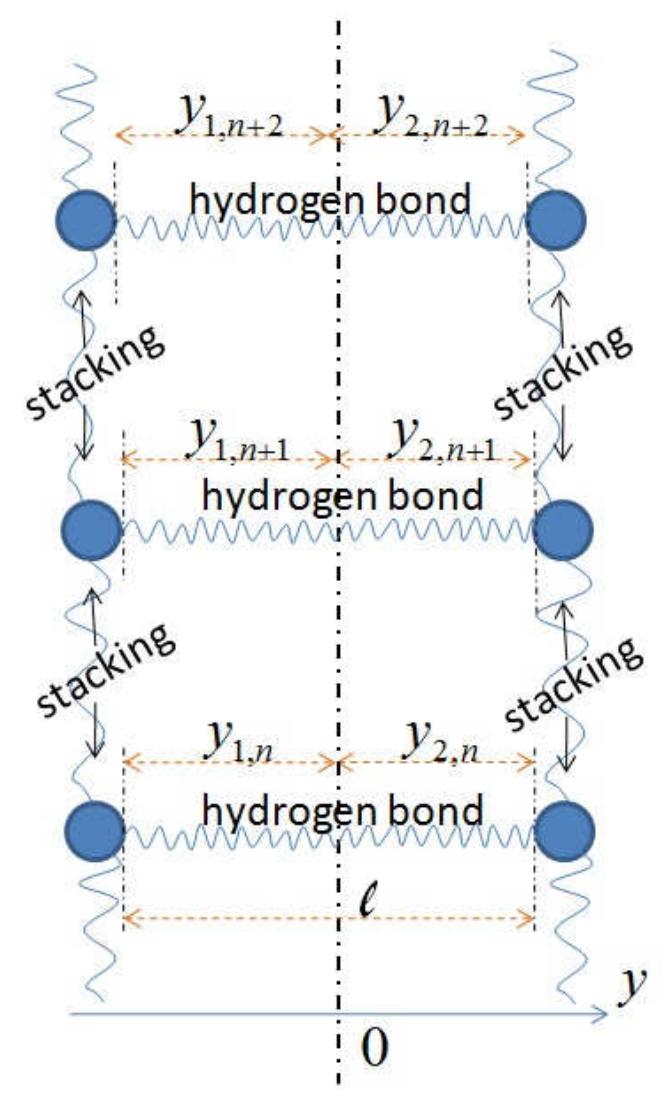

Figure 2: DNA sequence: double - stranded model.

\section{Results and discussion}

The charge localization in the DNA bases should be followed by local DNA lesions. Some assumptions about the nature of the lesions can be made if we consider the dynamics of Shift = $y_{1, n}-y_{2, n}+l$ of the base pairs. The case Shift $=0 \AA$ corresponds to the base equilibrium at a distance of $7 \AA$, the difference $y_{1, n}-y_{2, n}=-7 \AA$. A deviation from equilibrium (Shift $\neq 0 \AA$ ) should result in local DNA disruption.

Figure. 3 a) shows the structure of $T_{1} A_{2} G_{3} G(+) C_{5} G_{6} A_{7} G_{8} T_{9}$ sequence, where the index corresponds to the base position in the sequence under consideration and $G(+)$ corresponds to the charge localization in the initial moment of time. The charge localization on the DNA base sequence leads to the deformation of the latter. Figure. $3 \mathrm{~b}$ ) shows time dependencies of the base-pair displacement. The numbers correspond to the position of the bases-pair in the sequence.

Consider the pair $G(+)-C$ where the charge was localized at the initial moment of time. The model takes into account the fact that a positive charge makes the $G(+)-C$ pair become displaced, since the local structure of the pair is changed due to a missing electron, which apparently should lead to the DNA structure deforming. Thus, it is assumed that the first $G(+)-C$ pair is displaced horizontally from equilibrium at the initial moment of time. We assumed that the bases of the first $G(+)-C$ pair had the coordinates $y_{1, n}=-3.20 \AA$ and $y_{2, n}=4.00 \AA$. In other words, the bases of the pair, on one of which a charge was located, were at a distance $7.2 \AA$ from each other at the initial 


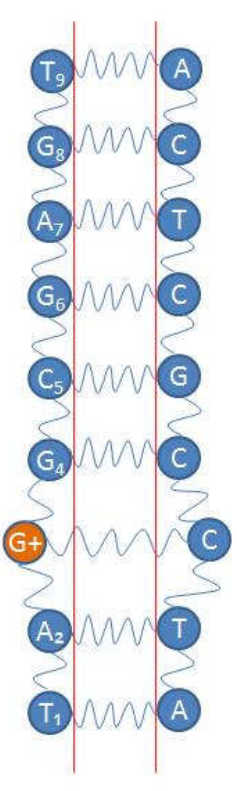

a)

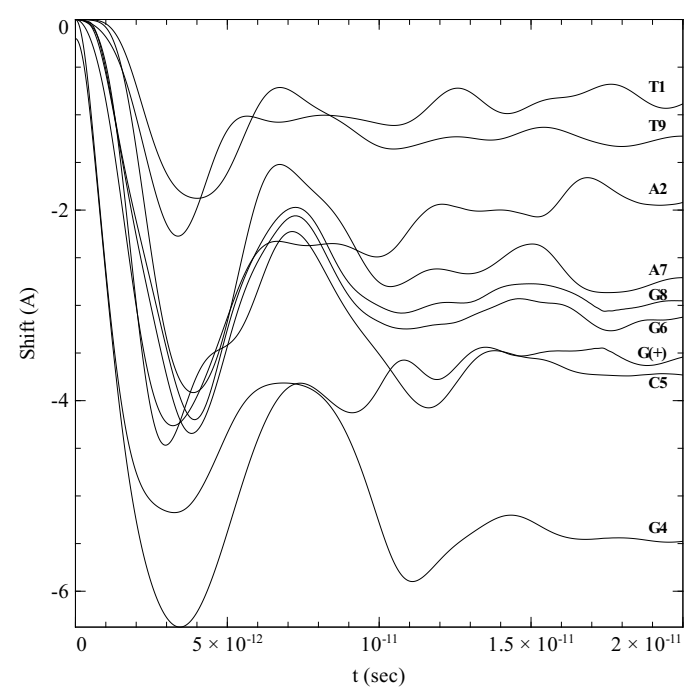

b)

Figure 3: a) DNA base sequence TAGGCGAGT, stretching of base pairs of DNA sequence. b) The dynamics of the base pairs Shift.

moment of time. In this case the first base pair $G(+)-C$ is shifted by $-0.2 \AA$ from equilibrium (Shift $=-0.2 \AA$ ) while the shift is equal to 0 for all the rest base pairs of the strand.

Then, the process stabilizes. The charge migrates from the first guanine $G(+)$, as a result the bases of the first pair $G(+)-C$ shift from equilibrium. At the moment of separation the charge physically acts on the base of the pair. The hole jumps out from the base. As a result the base pair begins to be swung. At the following moments of time, the process stabilizes at a Shift $=$ $-3.76 \AA$ suggesting a possible hydrogen bond $G(+)-C$ disruption. The charge migration process is accompanied by the significant deviation of $G 4-C$ pair from equilibrium position (Shift $=$ $5.75 \AA$ ). In virtue of stacking bonds, the shift of the pairs under consideration should be followed by that of their neighboring pairs. That is why the deviation of the $C 5-G$ pair is not zero. A considerable shift difference between the pairs suggests some possible one and two-strand lesions between them. A considerable shift difference between the pairs $G(+)-C$ and $G 4-C, G 8-C$ and $T 9-A$ pairs, $T 1-A$ and $A 2-T$ pairs suggests some possible disruptions between them. However since a difference in the displacements between pairs $G(+)-C$ and $G 4-C, G 8-C$ and $T 9-A$ are most great the break in these sections it is most probable. As a result $G 3-$ and $G 4-, G 8-C$ and $T 9-A$ pairs dropping out of the strand.

\section{References}

[1] Gomez-Tejedor G.G. and Fuss M.Ch. Radiation Damage in Biomolecular Systems. London: Springer, 2012. 